\title{
EIGENFUNCTION EXPANSION FOR A REGULAR FOURTH ORDER EIGENVALUE PROBLEM WITH EIGENVALUE PARAMETER IN THE BOUNDARY CONDITIONS
}

\section{E.M.E. ZAYED}

Department of Mathematics

Faculty of Science

Zagazig University

Zagazig, Egypt

and

\section{S.F.M. IBRAHIM}

Department of Mathematics

Faculty of Education

Ain Shams University

Helipolis, Calro, Egypt

(Rece Ived October 15, 1987)

1. INTRODUCTION.

The regular right-definite efgenvalue problems for second order differential equations with elgenvalue parameter in the boundary conditions, have been studied in Wa1ter [1], Fulton [2] and Hinton [3].

The object of this paper is to prove the expansion theorem for the following regular fourth order eigenvalue problem:

$$
\left.\begin{array}{rl}
\pi u: \quad & \left(K u^{\prime \prime}\right)^{\prime \prime}-\left(P u^{\prime}\right)^{\prime}+q u=\lambda u, x \varepsilon[a, b] \\
& u(a)=\left(P u^{\prime}\right)(a)=\left(K u^{\prime \prime}\right)(a)=0 \\
& \left(K u^{\prime \prime \prime}\right)(b)-\left(P u^{\prime}\right)(b)=-\lambda u(b)
\end{array}\right\}
$$

where $P, q$ and $K$ are continuous real-valued functions on $[a, b]$. We assume that $P(x)>0, q(x)>0$, and $K(x)>0$ while $\lambda$ is a complex number.

Recently, Zayed [4] has studied the special case of the problem (1.1) wherein $K(x)=\alpha^{2}, \quad \alpha^{2}$ is a constant and $q(x)=0$.

Further, problem (1.1), in general, describes the transverse motion of a rotating beam with tip mass, such as a helicopter blade (Ahn [5]) or a bob pendulum suspended from a wire (Ahn [6]). 
Ahn [7] has shown that the set of elgenvalues of problem (1.1) is not empty, has no finite accumulation points and is bounded from below. He used an integral-equation a pproach.

In this paper, our approach is to give a Hilbert space formulation to the problem (1.1) and self-adjoint operator defined in it such that (1.1) can be considered as the efigenvalue problem of this operator.

2. HILBERT SPACE FORMULATION.

We define a Hilbert space $H$ of two-component vectors by

$$
H=L^{2}(a, b) \oplus C ;
$$

with inner product

and norm

$$
\langle f, g\rangle=\int_{a}^{b} f_{1} \bar{g}_{1} d x+f_{2} \bar{g}_{2} \quad, f, g \in H
$$

$$
\|\left. f\right|_{H} ^{2}=\int_{a}^{b}\left|f_{1}\right|^{2} d x+\left|f_{2}\right|^{2}
$$

where

and

$$
f=\left(f_{1}, f_{2}\right)=\left(f_{1}(x), f_{1}(b)\right) \varepsilon H
$$

$$
g=\left(g_{1}, g_{2}\right)=\left(g_{1}(x), g_{1}(b) \varepsilon H\right. \text {. }
$$

We can define a linear operator $A: D(A) \rightarrow H$ by

$$
\text { Af }=\left(\tau f_{1},-\left(K f_{1}^{\prime \prime}\right)(b)+\left(P f_{1}^{\prime}\right)(b)\right) \quad \forall f=\left(f_{1}, f_{2}\right) \varepsilon D(A)
$$

where the domain $D(A)$ of $A$ is a set of all $f=\left(f_{1}, f_{2}\right) \varepsilon$ which satisfy the following:

(1) $f_{1}, f_{1}^{\prime}, f_{1}^{\prime \prime}$ and $f_{1}^{\prime \prime}$ are absolutely continuous with

$$
\mathrm{If}_{1} \varepsilon L^{2}(a, b) \text { and } \int_{a}^{b}\left(K\left|f_{1}^{\prime \prime}\right|^{2}+P\left|f_{1}^{\prime}\right|^{2}+q\left|f_{1}\right|^{2}\right) d x<\infty \text {. }
$$$$
\text { (i1) } f_{1}(a)=\left(P f_{1}^{\prime}\right)(a)=\left(K f_{1}^{\prime \prime}\right)(a)=0
$$$$
\text { (iii) } f_{2}=f_{1}(b)
$$

REMARK 2.1. The parameter $\lambda$ is an eigenvalue of (1.1) and $f_{1}$ is a corresponding eigenfunction of $(1.1)$ if and only if

$$
f=\left(f_{1}, f_{1}(b)\right) \varepsilon D(A) \quad \text { and } \quad \text { Af }=\lambda f
$$

Therefore, the efgenvalues and the eigenfunctions of problem (1.1) are equivalent to the eigenvalues and the eigenfunctions of operator A. 
We consider the following assumptions:

$$
\begin{aligned}
& \text { (i) } \lim _{x \rightarrow b}\left[K^{\prime}(x) f_{1}(x)-K(x) f_{1}^{\prime}(x)\right]=0, \\
& \text { (ii) } \lim _{x \rightarrow b}\left[K^{\prime}(x) \bar{g}_{1}(x)-K(x) \bar{g}_{1}^{\prime}(x)\right]=0 .
\end{aligned}
$$

LEMMA 2.1. The linear operator $A$ in $H$ is symmetric.

PROOF. On using the boundary conditions of (1.1) we get,

$$
\begin{aligned}
\langle A f, g\rangle & =\int_{a}^{b}(\tau f) \bar{g}_{1} d x+\left[-\left(K f_{1}^{\prime \prime}\right)(b)+\left(P f_{1}^{\prime}\right)(b)\right] \bar{g}_{1}(b) \\
& =\int_{a}^{b}\left(K f_{1}^{\prime \prime}\right) \bar{g}_{1} d x-\int_{a}^{b}\left(P f_{1}^{\prime}\right) \bar{g}_{1} d x+\int_{a}^{b} q f_{1} \bar{g} d x-\left(K f_{1}^{\prime}\right)(b) \bar{g}_{1}(b) \\
& +\left(P f_{1}^{\prime}\right)(b) \bar{g}_{1}(b)
\end{aligned}
$$

Integrating the first term of $(2.6)$ by parts four times and integrating the second term of (2.6) by parts twice, we get

$$
\begin{aligned}
\langle A f, g\rangle & =\int_{a}^{b} f_{1}\left[\left(\overline{K g}_{1}^{\prime \prime}\right)^{\prime \prime}-\left(\overline{P g}_{1}^{\prime}\right)++\overline{q g}_{1}\right] d x+f_{1}(b)\left[-\left(\overline{K g}_{1}^{\prime \prime}\right)(b)+\left(\overline{P g}_{1}^{\prime}\right)(b)\right] \\
& +f_{1}^{\prime \prime}(b)\left[K^{\prime}(b) \bar{g}_{1}(b)-K(b) \bar{g}_{1}^{\prime}(b)\right]-\bar{g}_{1}^{\prime \prime}(b)\left[K^{\prime}(b) f_{1}(b)-K(b) f_{1}^{\prime}(b)\right]
\end{aligned}
$$

Applying the conditions (2.5) and using the boundary conditions of (1.1), we obtain

$$
\left.\left.\langle A f, g\rangle=\int_{a}^{b} f_{1}\left(\overline{\tau g_{1}}\right) d x+f_{1}(b)\left[-\overline{(K g}_{1}^{\prime \prime \prime}\right)(b)+\bar{P}_{g_{1}^{\prime}}\right)(b)\right]=\langle f, A g\rangle .
$$

REMARK. 2.2. For all $f=\left(f_{1}, f_{2}\right)$ in $D(A)$ and $f_{2}=f_{1}(b) \neq 0$, the domain $D(A)$ is dense in $\mathrm{H}$.

Since the operator $A$ in $H$ is symmetric and dense in $H, A$ is self-adjoint.

\section{THE BOUNDEDNESS.}

We shall show that the self-adjoint operator $A$ is unbounded from above and bounded from below. We also show that A is strictly positive.

LEMMA 3.1 .

(1) If $f, f^{\prime}$ are absolutely continuous with $f(a)=0$ and $P(x)>0$ in $[a, b]$, then we have $P(x)>c_{1}$ for some constant $c_{1}>0$ such that

$$
\int_{a}^{b} P(x)\left|f^{\prime}(x)\right|^{2} d x>c_{1}|f(b)|^{2}
$$

(ii) For $f \varepsilon C^{2}[a, b]$, there exists a positive constant $c_{2}$ such that

$$
\int_{a}^{b}|f(x)|^{2} d x<c_{2} \int_{a}^{b}\left|f^{\prime \prime}(x)\right|^{2} d x
$$


PROOF.

(1) Since $P(x)>0$ in $[a, b]$, we have $P(x)>c_{1}$ for some $c_{1}>0$. Consequently, on using Schwartz's inequality, we get

$$
\int_{a}^{b} P(x)\left|f^{\prime}(x)\right|^{2} d x>c_{1} \int_{a}^{b}\left|f^{\prime}(x)\right|^{2} d x>c_{1}\left[\int_{a}^{b}\left|f^{\prime}(x)\right| d x\right]^{2}>c_{1}|f(b)|^{2}
$$

where $\int_{a}^{b} f^{\prime}(x) d x=f(b)-f(a)=f(b)$, Since $f(a)=0$.

(1i) By using Theorem 2 in $[8, p .67]$, we have for $f(x) \varepsilon C^{1}[a, b]$,

$$
\int_{a}^{b}|f(x)|^{2} d x<4(b-a)^{2} \int_{a}^{b}\left|\frac{d|f(x)|}{d x}\right|^{2} d x
$$

Since $\quad\left|\frac{d|f(x)|}{d x}\right|^{2}<4\left|\frac{d f(x)}{d x}\right|^{2}$,

then

$$
\int_{a}^{b}|f(x)|^{2} d x<4(b-a)^{2} \int_{a}^{b} \frac{\left.|d| f(x)\right|^{2}}{d x} d x<16(b-a)^{2} \int_{a}^{b}\left|f^{\prime}(x)\right|^{2} d x
$$

Applying (3.1) again for $\left|f^{\prime}(x)\right|$, we get

$$
\int_{a}^{b}\left|f^{\prime}(x)\right|^{2} d x<16(b-a)^{2} \int_{a}^{b}\left|f^{\prime \prime}(x)\right|^{2} d x
$$

from (3.1) and (3.2) we get

$$
\int_{a}^{b}|f(x)|^{2} d x<c_{2} \int_{a}^{b}\left|f^{\prime \prime}(x)\right|^{2} d x \text { where the constant } c_{2}=256(b-a)^{4} \text {. }
$$

LEMMA 3.2. The linear operator $A$ is bounded from below.

PROOF. On using the boundary conditions of $(1.1)$ we get

$$
\begin{aligned}
\langle A f, f\rangle & =\int_{a}^{b}\left(\tau f_{1}\right) \bar{f}_{1} d x+\left[-\left(K f_{1}^{\prime \prime}\right)(b)+\left(P f_{1}^{\prime}\right)(b)\right] \bar{f}_{1}(b) \\
& =\int_{a}^{b}\left(K f_{1}^{\prime \prime}\right) \bar{f}_{1} d x-\int_{a}^{b}\left(P f_{1}^{\prime}\right) \bar{f}_{1} d x+\int_{a}^{b} q f_{1} \bar{f}_{1} d x-\left(K f_{1}^{\prime \prime}\right)(b) \bar{f}_{1}(b) \\
& +\left(P f_{1}^{\prime}\right)(b) \bar{f}_{1}(b) .
\end{aligned}
$$

Integrating (3.3) by parts twice and using the boundary conditions of (1.1), we obtain

$$
\begin{aligned}
\langle A f, f\rangle & =f_{1}^{\prime \prime}(b)\left[K^{\prime}(b) \bar{f}_{1}(b)-K(b) \bar{f}_{1}^{\prime}(b)\right]+\int_{a}^{b} K\left|f_{1}^{\prime}\right|^{2} d x \\
& +\int_{a}^{b} P\left|f_{1}\right|^{2} d x+\int_{a}^{b} q\left|f_{1}\right|^{2} d x
\end{aligned}
$$

On using (2.5) (1i) and lemma (3.1), we get

$$
\begin{aligned}
\langle A f, f\rangle & >\int_{a}^{b} \frac{k(x)}{c_{2}}\left|f_{1}(x)\right|^{2} d x+c_{1}\left|f_{1}(b)\right|^{2}+\int_{a}^{b} q(x)\left|f_{1}(x)\right|^{2} d x \\
& =\int_{a}^{b}\left[\frac{K(x)}{c_{2}}+q(x)\right]\left|f_{1}(x)\right|^{2} d x+c_{1}\left|f_{2}\right|^{2}
\end{aligned}
$$


where

$$
>c_{3} \int_{a}^{b}\left|f_{1}(x)\right|^{2} d x+c_{1}\left|f_{2}\right|^{2}
$$

Therefore

$$
c_{3}=\operatorname{lnf}_{x \varepsilon[a, b]}\left[\frac{K(x)}{c_{2}}+q(x)\right]
$$

$$
\langle\text { Af, } \mathrm{f}\rangle>\mathrm{c}\|\mathrm{f}\|^{2}
$$

where the constant $c=\min \left(c_{3}, c_{1}\right)$.

It follows, from (3.4), that the operator $A$ is bounded from below. Since $c_{1}>0, \mathrm{~K}(\mathrm{x})>0, \mathrm{q}(\mathrm{x})>0, \mathrm{c}_{2}>0$ and $\mathrm{c}=\min \left(\mathrm{c}_{3}, \mathrm{c}_{1}\right)$ then the constant $\mathrm{c}$ is positive $(c>0)$ and hence $A$ is strictly positive.

REMARK 3.1.

(1) Since A is a symmetric operator (from lemma 2.1) then A has only real e igenva lues.

(11) By Lemma 3.2, we deduce that the set of all eigenvalues of $A$ is also bounded from below.

(1i1) Since $A$ is strictly positive, then the zero is not an eigenvalue of A.

By using theorem 3 in $[8, p .60]$ we can state that:

Since $A$ in $H$ is symmetric and bounded from below, then for every elgenvalue $\lambda_{1}$ of A in $H, \lambda_{1}>c$ where the constant $c$ is the same as in (3.4). This means that $0<c<\lambda_{1}<\lambda_{2}<\ldots \ldots \ldots \ldots<\lambda_{1}$ according to the size and $\lambda_{1}+\infty$ as $1+\infty$. This implies that the set of all eigenvalues of $A$ is unbounded from above.

REMARK 3.2. Since the operator $A$ is self-adjoint, then $A$ has only real eigenvalues and the eigenfunctions of $A$ are orthonormal. By using theorem 3 in [8, p.30], the density of the domain $D(A)$ in $H$ gives us the completeness of the orthonormal system of elgenfunctions $\Phi_{1}, \Phi_{2}, \Phi_{3}, \ldots$ of $A$.

4. THE EIGENFUNCTIONS OF THE OPERATOR A.

We suppose $\phi_{\lambda}(x), \Psi_{\lambda}(x), x_{\lambda}(x)$ and $\gamma_{\lambda}(x)$, where $\lambda \in C$ is not an eigenvalue of $A$, are the fundamental set of solutions of the fourth order differential equation of (1.1) with the initial conditions:

$$
\begin{array}{llll}
\phi_{\lambda}(\mathrm{a})=0, & \left(\mathrm{P} \phi_{\lambda}^{\prime}\right)(\mathrm{a})=0, & \phi_{\lambda}^{\prime \prime}(\mathrm{a})=1, & \left(\mathrm{~K} \phi_{\lambda}^{\prime \prime \prime}\right)(\mathrm{a})=0 \\
\psi_{\lambda}(\mathrm{a})=0, & \left(\mathrm{P} \psi_{\lambda}^{\prime}\right)(\mathrm{a})=0, & \psi_{\lambda}^{\prime \prime}(\mathrm{a})=0, & \left(\mathrm{~K} \psi_{\lambda}^{\prime \prime \prime}\right)(\mathrm{a})=1 \\
\chi_{\lambda}(\mathrm{b})=0, & \left(\mathrm{P}_{\lambda}^{\prime}\right)(\mathrm{b})=1, & \chi_{\lambda}^{\prime \prime}(\mathrm{b})=0, & \left(\mathrm{~K}_{\chi_{\lambda}^{\prime}}^{\prime \prime \prime}\right)(\mathrm{b})=1 \\
\gamma_{\lambda}(\mathrm{b})=1, & \left(\mathrm{P}_{\lambda}^{\prime}\right)(\mathrm{b})=1+\lambda, & \gamma_{\lambda}^{\prime \prime}(\mathrm{b})=0, & \left(\mathrm{~K}_{\lambda}^{\prime \prime \prime}\right)(\mathrm{b})=1
\end{array}
$$

Therefore the Wronskian is

$$
W=\lim _{x \rightarrow b}\left[x_{\lambda}(x)\left(P Y_{\lambda}^{\prime}\right)(x)-\left(P x_{\lambda}^{\prime}\right)(x) Y(x)\right]=-1 \neq 0
$$


Thus the solutions $\phi_{\lambda}(x), \psi_{\lambda}(x), \chi_{\lambda}(x)$ and $\gamma_{\lambda}(x)$ a re linearly independent of $\tau u=\lambda u$. Putting $x=b$, we obtain the Wronskian in the form:

$$
\begin{aligned}
W & =\psi_{\lambda}^{\prime \prime}(b)\left[\lambda \phi_{\lambda}(b)-\left(P \phi_{\lambda}^{\prime}\right)(b)+\left(K \phi_{\lambda}^{\prime \prime \prime}\right)(b)\right] \\
& -\phi_{\lambda}^{\prime \prime}(b)\left[\lambda \psi_{\lambda}(b)-\left(P \psi_{\lambda}^{\prime}\right)(b)+\left(K \psi_{\lambda}^{\prime \prime \prime}\right)(b)\right] \neq 0
\end{aligned}
$$

Now, for $f=\left(f_{1}, f_{2}\right) \in H$, we define $\Phi=\left(\Phi_{1}, \Phi_{2}\right) \in D(A)$ as the unique solution of $(\lambda I-A) \Phi=f$.

Application of variation of parameter method ylelds the unique solution $\Phi \varepsilon D(A)$ of $(\lambda I-A) \Phi=f$, f $\varepsilon H$ with:

The refore

$$
\begin{aligned}
& (\lambda I-\tau) \Phi_{1}=f_{1} \\
& \lambda \Phi_{1}(b)-\left(P \Phi_{1}^{\prime}\right)(b)+\left(K \Phi_{1}^{\prime \prime}\right)(b)=f_{2}
\end{aligned}
$$

$$
\begin{aligned}
\Phi_{1}(x) & =\int_{a}^{b}\left[\frac{\phi_{\lambda}(x) \alpha_{1}(t)+\psi_{\lambda}(x) \alpha_{2}(t)}{W}\right] f_{1}(t) d t \\
& +\int_{a}^{b}\left[\frac{x_{\lambda}(x) \alpha_{3}(t)+\gamma_{\lambda}(x) \alpha_{4}(t)}{W}\right] f_{1}(t) d t \\
& +d_{1} \phi_{\lambda}(x)+d_{2} \psi_{\lambda}(x)+d_{3} x_{\lambda}(x)+d_{4} \gamma_{\lambda}(x),
\end{aligned}
$$

where

and

$$
\begin{aligned}
& \alpha_{1}(t)=\frac{-P(t)}{K(t)}\left|\begin{array}{lll}
\psi_{\lambda}(t) & x_{\lambda}(t) & \gamma_{\lambda}(t) \\
\psi_{\lambda}^{\prime}(t) & x_{\lambda}^{\prime}(t) & \gamma_{\lambda}^{\prime}(t) \\
\psi_{\lambda}^{\prime \prime}(t) & \chi_{\lambda}^{\prime \prime}(t) & \gamma_{\lambda}^{\prime \prime}(t)
\end{array}\right| \\
& \alpha_{2}(t)=\frac{P(t)}{K(t)}\left|\begin{array}{lll}
\phi_{\lambda}(t) & x_{\lambda}(t) & \gamma_{\lambda}(t) \\
\phi_{\lambda}^{\prime}(t) & x_{\lambda}^{\prime}(t) & \gamma_{\lambda}^{\prime}(t) \\
\phi_{\lambda}^{\prime \prime}(t) & \chi_{\lambda}^{\prime \prime}(t) & \gamma_{\lambda}^{\prime \prime}(t)
\end{array}\right| \\
& \alpha_{3}(t)=\frac{-P(t)}{K(t)}\left|\begin{array}{lll}
\phi_{\lambda}(t) & \psi_{\lambda}(t) & \gamma_{\lambda}(t) \\
\phi_{\lambda}^{\prime}(t) & \psi_{\lambda}^{\prime}(t) & \gamma_{\lambda}^{\prime}(t) \\
\phi_{\lambda}^{\prime \prime}(t) & \psi_{\lambda}^{\prime \prime}(t) & \gamma_{\lambda}^{\prime \prime}(t)
\end{array}\right|
\end{aligned}
$$

$$
\alpha_{4}(t)=\frac{P(t)}{K(t)} \quad\left|\begin{array}{lll}
\phi_{\lambda}(t) & \psi_{\lambda}(t) & x_{\lambda}(t) \\
\phi_{\lambda}^{\prime}(t) & \psi_{\lambda}^{\prime}(t) & x_{\lambda}^{\prime}(t) \\
\phi_{\lambda}^{\prime \prime}(t) & \psi_{\lambda}^{\prime \prime}(t) & x_{\lambda}^{\prime \prime}(t)
\end{array}\right|
$$

while $d_{1}, d_{2}, d_{3}$ and $d_{4}$ a re constants.

Calculation of $\Phi_{1}(b), \Phi_{1}^{\prime}(b)$ and $\Phi_{1}^{\prime \prime}(b)$ from (4.7) and substitution into (4.6) with the initial conditions $(4.3)$ and $(4.4)$, we can get the constants $d_{1}, d_{2}, d_{3}$ and $d_{4}$ as follows: 
and $d_{3}=d_{4}=0$.

$$
\begin{aligned}
& d_{1}=\frac{1}{W}\left[-f_{2} \psi_{\lambda}^{\prime \prime}(b)+\int_{a}^{b} q_{1}(t) f_{1}(t) d t\right], \\
& d_{2}=\frac{1}{W}\left[f_{2} \phi_{\lambda}^{\prime \prime}(b)+\int_{a}^{b} q_{2}(t) f_{1}(t) d t\right]
\end{aligned}
$$

Consequently, we deduce that

and

$$
\Phi_{1}(x)=\frac{f_{2}}{W}\left[\psi_{\lambda}(x) \phi_{\lambda}^{\prime \prime}(b)-\phi_{\lambda}(x) \psi_{\lambda}^{\prime \prime}(b)\right]+\int_{a}^{b} G(x, t, \lambda) f_{1}(t) d t
$$

$$
\Phi_{2}=\Phi_{1}(\mathrm{~b})
$$

where $G(x, t, \lambda)$ is the Green's function defined by:

$$
\frac{\phi_{\lambda}(x) \alpha_{1}(t)+\psi_{\lambda}(x) \alpha_{2}(t)}{W}
$$

$$
G(x, t, \lambda)=
$$

$$
\frac{x_{\lambda}(x) \alpha_{3}(t)+\gamma_{\lambda}(x) \alpha_{4}(t)}{W}
$$$$
a<t<x<b
$$

The form of equations (4.8) and (4.9) shows that the inverse operator $(\lambda I-A)^{-1}$ is actually compact; for details of argument of theorem 5 in $[8, p .120]$ can be used.

\section{EXPANSION THEOREM.}

We now arrive at the problem of expanding an arbitrary function $f(x) \varepsilon H$ for $x \varepsilon[a, b]$ in terms of the elgenfunctions of (1.1). The results of our ivestigations are summarized in the following theorem:

THEOREM 5.1. The operator $A$ in $H$ has unbounded set of real efgenvalues of finite multiplicity, (they have at most multiplicity four), without accumulation points In $(-\infty, \infty)$ and they can be ordered according to the size, $0<c<\lambda_{1}<\lambda_{2}<\ldots<\lambda_{1}$ with $\lambda_{1}+\infty$ as $1 \rightarrow \infty$. If the corresponding elgenfunctions $\Phi_{1}, \Phi_{2}, \Phi_{3}, \ldots$ form a complete orthonormal system, then for any function $f(x) \varepsilon H$, we have the expansion:

$$
f(x)=\sum_{1=1}^{\infty}\left\langle f, \Phi_{1}\right\rangle \Phi_{1}
$$

which is a uniformly convergent series.

The above theorem has some interesting corollarles for particular cholces of $\mathrm{f}$. COROLLARY 4.1. If $f_{1} \varepsilon L^{2}(a, b)$ and $f=\left(f_{1}, 0\right) \varepsilon H$, then we have

(i) $\quad f_{1}=\sum_{i=1}^{\infty}\left(\int_{a}^{b} f_{1} \Phi_{i 1} d x\right) \Phi_{i 1}(x)$

(ii) $0=\sum_{1=1}^{\infty}\left(\int_{a}^{b} f_{1} \Phi_{11} d x\right) \Phi_{12}$ 
COROLLARY 4.2. If $\Phi_{1}=\left(\Phi_{11}(x), \Phi_{12}\right) \varepsilon D(A)$ and $f=(0,1) \varepsilon H$, we have:

(i) $\quad 0=\sum_{i=1}^{\infty} \Phi_{i 2} \Phi_{i 1}(x)=\sum_{i=1}^{\infty} \Phi_{i 1}$ (b) $\Phi_{i 1}(x)$.

(i1) $1=\sum_{1=1}^{\infty}\left[\Phi_{12}\right]^{2}=\sum_{i=1}^{\infty}\left[\Phi_{11}(\mathrm{~b})\right]^{2}$.

\section{REFERENCES}

1. WALTER, J., Regular eigenvalue problems with elgenvalue parameter in the boundary condition, Math. Z. 133, (1973), 301-312.

2. FULTON, C.T., Two-point boundary value problems with elgenvalue parameter contained in the boundary conditions, Proc. Royal Soc. Edinburgh 77A, (1977), 293-308.

3. HINTON, D.B., An expansion theorem for an eigenvalue problem with eigenvalue parameter In the boundary condition, Quart. J. Math. Oxford 2, (1979), 33-42.

4. ZAYED, E.M.E., Regular elgenvalue problem with eigenvalue parameter in the boundary conditions, Proc. Math. Phys. Soc. Egypt 58, (1984), 55-62.

5. AHN, H.J., On random transverse vibrations of a rotating beam with tip mass, Q.J. Mech. Appl. Math. 36, (1983), 97-109.

6. AHN, H.J., Vibrations of a pendulum consisting of a bob suspended from a wire, Q. App1. Math. 39, (1981), 109-117.

7. AHN, H.J., Vibrations of a pendulum consisting a bob suspended from a wire. The method of Integral equations, Not. Am. Math. 26(1), (1979), A-75.

8. HELLWIG, G., Differential operators of Mathematical physics, Addison-Wesely Pub. Com., U.S.A., 1967. 


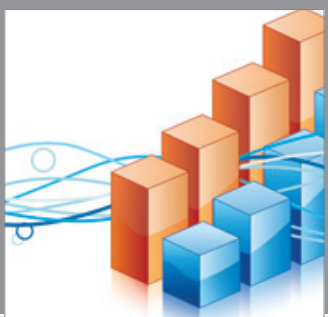

Advances in

Operations Research

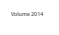

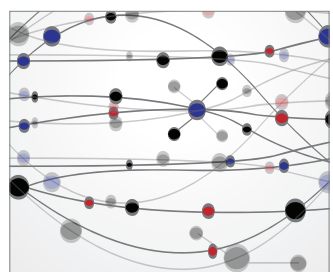

\section{The Scientific} World Journal
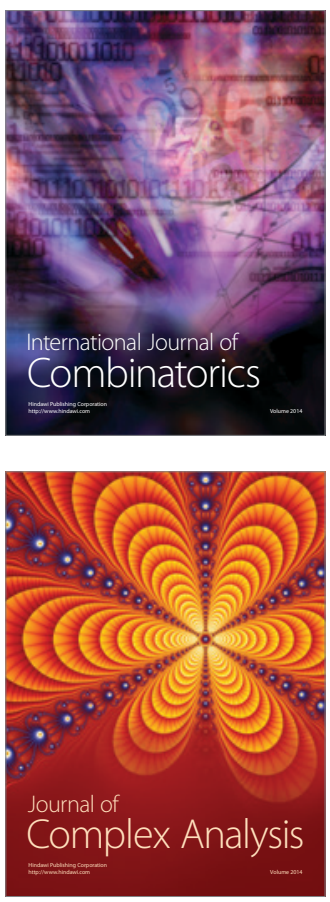

International Journal of

Mathematics and

Mathematical

Sciences
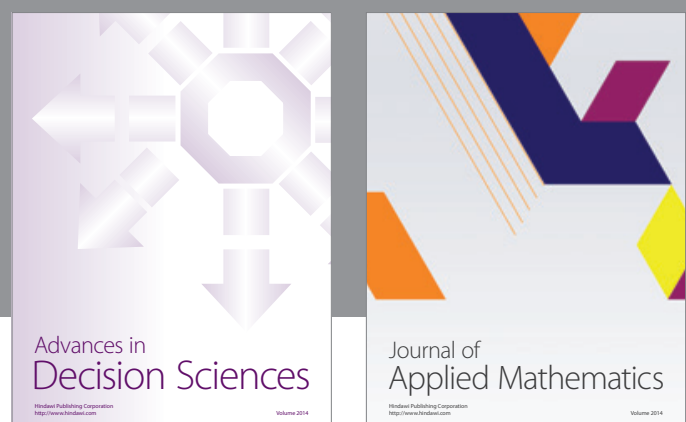

Journal of

Applied Mathematics
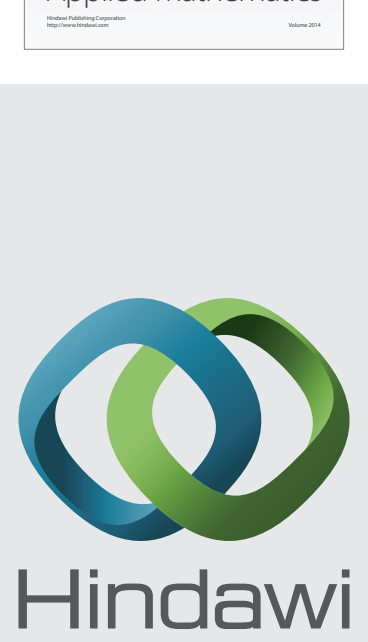

Submit your manuscripts at http://www.hindawi.com
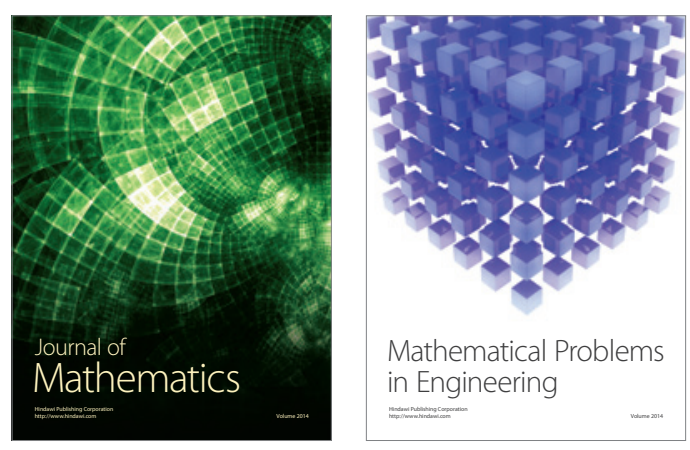

Mathematical Problems in Engineering
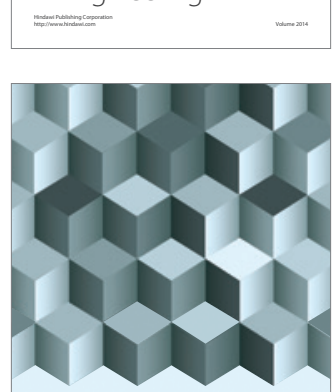

Journal of

Function Spaces
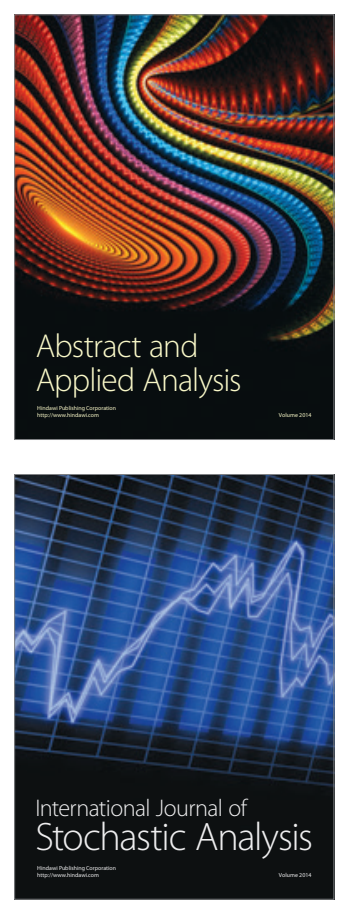

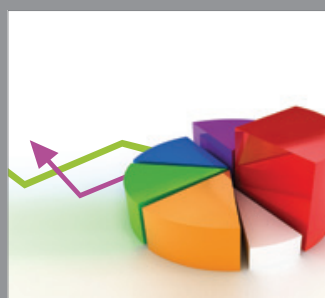

ournal of

Probability and Statistics

Promensencen
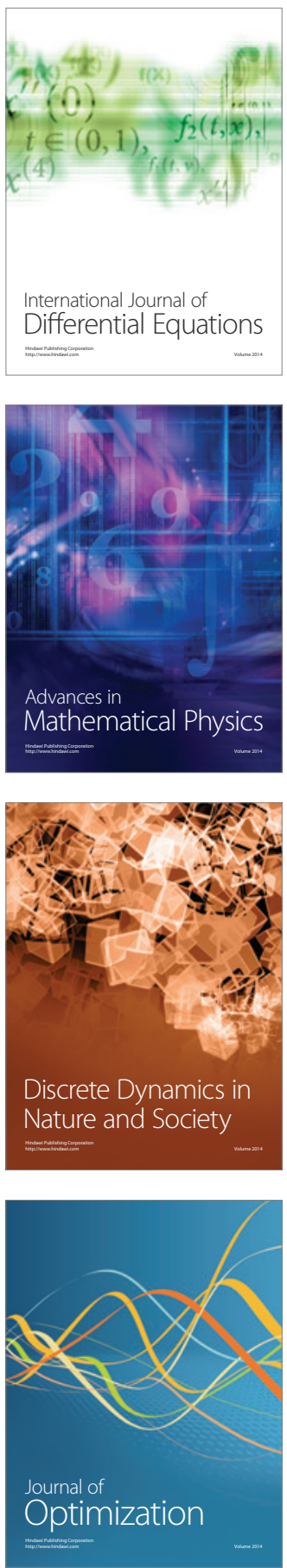